\title{
The use of ultrasound guided erector spinae plane block to optimize anesthetic regimen and improve patient satisfaction for open thoracic surgery: a randomized controlled clinical study
}

\section{Qiang Wang}

National Cancer Center/National Clinical Research Center for Cancer/Cancer Hospital, Chinese Academy of Medical Sciences and Peking Union Medical College

\section{Shuai Li}

National Cancer Center/National Clinical Research Center for Cancer/Cancer Hospital, Chinese Academy of Medical Sciences and Peking Union Medical College

\section{Shijing Wei}

National Cancer Center/National Clinical Research Center for Cancer/Cancer Hospital, Chinese Academy of Medical Sciences and Peking Union Medical College

Guo-Hua Zhang ( $\sim$ d1974@163.com)

National Cancer Center https://orcid.org/0000-0001-5039-4181

\section{Yong Li}

National Cancer Center/National Clinical Research Center for Cancer/Cancer Hospital, Chinese Academy of Medical Sciences and Peking Union Medical College

Hui Zheng

National Cancer Center/National Clinical Research Center for Cancer/Cancer Hospital, Chinese Academy of Medical Sciences and Peking Union Medical College

\section{Research article}

Keywords: Open thoracic surgery, Ultrasound guided erector spinae plane block, General anesthesia, Opioid consumption, Patient satisfaction

Posted Date: April 2nd, 2019

DOl: https://doi.org/10.21203/rs.2.1746/v1

License: (c) (i) This work is licensed under a Creative Commons Attribution 4.0 International License. Read Full License 


\section{Abstract}

Background: The opioid-related side effects exist extensively and have significant adverse effects on patients' recovery from general anesthesia and patient overall satisfaction after the thoracotomy.

Recently, the erector spinae plane block (ESPB) has been reported to be used as a new effective analgesia for thoracic neuropathic pain. This randomized study was designed to assess whether ultrasound guided ESPB could effectively reduce the intraoperative consumption of opioids, improve the quality of recovery, reduce postoperative adverse events and improve patient satisfaction. Methods: Sixty patients undergoing open thoracic surgery were allocated randomly into the following 2 groups: Combined ultrasound guided ESPB and general anesthesia (GA) group (group EG, $n=30$ ), and only GA group (group $G A, n=30$ ). In group $E G$, before general anesthetic induction, the ultrasound guided ESPB was performed at the level of the T5 transverse process $3 \mathrm{~cm}$ from the midline. Then, $25 \mathrm{ml}$ of $0.5 \%$ ropivacaine was injected deep to the erector spinae muscle. In both groups, the general anesthesia was inducted with 2 $\mathrm{mg} / \mathrm{kg}$ of propofol, $0.3 \mathrm{ug} / \mathrm{kg}$ of sufentanil, $0.6 \mathrm{mg} / \mathrm{kg}$ rocuronium. Then, the general anesthesia was maintained by inhaling 2.5\%-3.0\% sevoflurane (1.3-1.5 MAC) and intermittent administration of sufentanil and rocuronium. The intraoperative consumption of sufentanil, recovery time, tracheal extubation time, dermatomal levels of sensory blockade by ESPB, pain score at 60 minutes after surgery, consumption of rescue analgesic tramadol, postoperative adverse events and patient satisfaction with anesthetic regimen during 48 hours after the thoracotomy were all recorded. Results: The cutaneous sensory block of ESPB was from T2 to T9. Compared with group GA, the intraoperative sufentanil consumption, pain score, tramadol consumption, incidence of nausea and vomiting were all significantly less in group EG $(p<$ 0.05). In addition, the recovery time and tracheal extubation time were both significantly shorter in group EG compared to group GA $(p<0.001)$. And the patient satisfaction with anesthetic regimen in group EG was significantly higher compared to group GA $(p<0.001)$. Conclusions: Ultrasound guided ESPB combined with GA could significantly reduce intraoperative opioids consumption, provide a better recovery and improve patient satisfaction for open thoracotomy.

\section{Background}

At present, opioids are the most widely used intraoperative analgesics for surgeries, especially for stressful and painful major surgeries such as open thoracotomy[1, 2]. However, high dose of opioids results in severe nausea, vomiting, pruritus, constipation and even life-threatening complications such as respiratory depression. Thus, the use of regional nerve block technique, such as thoracic epidural block or paravertebral block, as an adjunct to general anesthesia is considered to be an effective option[3]. However, the potential risks related to thoracic epidural block, including epidural hematoma, epidural abscess, meningitis, spinal trauma, back pain and total spinal anesthesia make it less desirable for the multi-mode anesthesia, especially for patients with multiple complications[3, 4]. Similarly, as for paravertebral block, possible complications, such as pneumothorax, hematoma, nerve root injury, inadvertent spinal or epidural block still exist[3,5]. Thus, there continues to be growing interest in seeking more safer and simpler regional nerve block techniques for open thoracotomy. 
The erector spinae plane block (ESPB) is a novel truncal interfascial plane block, which is believed to target the dorsal and ventral rami of the thoracic spinal nerves[6]. In several recent case reports, by injection of local anesthetics deep into the erector spinae muscle, the ESPB could provide effect analgesia to treat thoracic neuropathic pain and acute postoperative pain after thoracic surgery[6, 7$]$. The significant advantage of ESPB is that, under the ultrasound guidance, it becomes a safe and simple regional nerve block technique. However, to our best knowledge, until now, no randomized, controlled, clinical study has been conducted to investigate the effects of combined ultrasound guided ESPB and general anesthesia on open thoracotomy. This randomized, controlled, clinical study was designed to observe the effects of combining ultrasound guided ESPB with general anesthesia on the intraoperative opioid consumption, quality of anesthesia recovery, postoperative analgesia, adverse postoperative events and patient satisfaction with anesthetic regimen for thoracotomy.

\section{Methods}

\section{Patients}

This single center, prospective, randomized, controlled clinical study was approved by the institutional ethics committee of National Cancer Center/National Clinical Research Center for Cancer/Cancer Hospital, Chinese Academy of Medical Sciences and Peking Union Medical College (NCC201804009), and registered on ClinicalTrials.gov (ChiCTR1800016583). The written informed consent was obtained from every patient. Sixty-two patients with lower thoracic esophageal cancer, American Society of Anesthesiologists (ASA) physical status I and II, ages 30 to 65 years old, and selected for open thoracic surgery (Sweet procedure) were recruited in this study. All patients were able to communicate well and understood how to evaluate their pain score and total satisfaction with the anesthesia regimen. In order to eliminate potential confounders from surgeons, all open thoracic surgeries were performed by only the same experienced surgical team ( $\mathrm{Li}$ Y, et al.). All patients were randomly allocated into the following two groups using a computer-generated random number table: group EG and group GA. In group EG, the ultrasound guided ESPB with $25 \mathrm{ml}$ of $0.5 \%$ ropivacaine was performed ipsilateral before the general anesthetic induction. Then the general anesthesia was inducted. In group GA, only general anesthesia was implemented. The anesthetics scheme of general anesthesia was same in both groups. Group allocation was concealed with the use of coded, opaque, sealed envelopes. Patients with infections at the site of injection for ultrasound guided ESPB; neuropathy; coagulation disorders; morbid obesity (body mass index $\geq 40 \mathrm{~kg} / \mathrm{m}^{2}$ ); allergy to ropivacaine; greater than first-degree heart block; bradycardia (heart rate $<60 \mathrm{bpm}$ ); pregnancy; clinically significant cardiovascular, pulmonary, hepatic or renal diseases; psychiatric illnesses that would interfere with assessment of pain score and satisfaction and those given a painkiller within one week before surgery were all excluded from this study. During preoperative interview, patients were taught how to evaluate their pain score by the numerical pain rating scale (NRS) and their own satisfaction with the anesthesia regimen by a 5 -point Likert scale $(5=$ completely satisfied, 4 = quite satisfied, 3 = slightly dissatisfied, 2 = dissatisfied, 1 = very dissatisfied). The flowchart of this study protocol was shown in Figure 1. 
Anesthesia, Monitoring, and Surgery

On arrival at the operating room, routine monitoring for open thoracotomy, including electrocardiography, invasive blood pressure, and pulse oxygen saturation $\left(\mathrm{SpO}_{2}\right)$, were applied. Then a double lumen (16gauge/16-gaunge) central venous catheter was placed by catheterization of right subclavian vein. The standardized anesthetic technique was used in the two groups. After anesthesia induction, a doublelumen endobronchial tube was placed guided by fiberoptic bronchoscopy. Then the patient was turned to a right lateral decubitus position from the supine position. The correct position of the double-lumen endobronchial tube was confirmed by fiberoptic bronchoscopy again. Before skin incision, one-lung ventilation was initiated. The volume-controlled ventilation mode was adopted, with the airway pressure and lung compliance monitored. General anesthesia was maintained with 2.5\%-3.0\% sevoflurane (1.3-1.5 MAC). The end-tidal carbon dioxide partial pressure was maintained between 35 to $45 \mathrm{~mm} \mathrm{Hg}$ by mechanical ventilation. During the surgery procedures, $0.2 \mu \mathrm{g} / \mathrm{kg}$ of sufentanil was administered intravenously in both groups when the systolic arterial pressure exceeded $120 \%$ of the preoperative value or the heart rate exceeded $100 \mathrm{bpm}$. This dose was repeated every 10 minutes until the blood pressure or heart rate returned to the required limits. And rocuronium was administrated as required. The consumption of sufentanil in each group during the general anesthesia was recorded.

After ipsilateral lung collapse, a posterolateral incision in the sixth intercostals space on the left side of the chest was made to dissociate the esophagus and clear the way to access the lymph nodes in the thoracic region. The diaphragm was opened for dissociation of the stomach to clear the way for access to the abdominal lymph nodes. The anastomosis was performed at the top of the thorax using an anastomat. At the conclusion of the surgical procedure, a chest drain was placed. At the end of the surgery, sevoflurane inhalation was stopped and the intravenous analgesic pump was applied. When the reversibility of muscle relaxant was sufficient and the physiological parameters were stable, the tracheal extubation was performed. Then, the recovery time from general anesthesia and the tracheal extubation time were all recorded.

Ultrasound guided erector spinae plane block

Before the general anesthesia induction, the patient was placed in a right lateral decubitus position and a high-frequency linear ultrasound transducer (GE LOGIQe, Wauwatosa, Wisconsin) was placed in a longitudinal orientation $3 \mathrm{~cm}$ lateral to the T5 spinous process. Then three muscles were identified superficial to the hyperechoic transverse process shadow as follows: trapezius, rhomboid major, and erector spinae. Under the ultrasound guided, an 8-cm 22-gauge block needle (Contiplex; B Braun, Melsungen, Germany) was inserted in-plane in a caudad-to-cephalad direction until the tip lay in the surface of the transverse process (Figure 2). Correct needle tip position was confirmed by visualizing linear fluid spread that separated the erector spinae muscle from the transverse process. Then, $25 \mathrm{ml}$ of 
$0.5 \%$ ropivacaine was injected deep to the erector spinae muscle (Figure 2 ). The assessment of cutaneous sensory block by pinprick was initiated 30 minutes later by a blind observer. After confirmation and assessment of the sensory block to pinprick, the general anesthesia induction was initiated.

Postanesthesia Care Unit and Postoperative Assessments

The patient was transferred to the postanesthesia care unit (PACU) after the operation. Then vital signs including heart rate, invasive blood pressure, respiratory rate, and $\mathrm{SpO}_{2}$ were monitored and recorded every 5 minutes for at least 60 minutes. A 20\% decrease in systolic blood pressure from baseline was defined as hypotension. Bradycardia was a condition defined wherein the heart rate was less than 60 bpm. Hypoxemia was a condition defined wherein the $\mathrm{SpO}_{2}$ was lower than $90 \%$ at room air. Hypotension was treated by $6 \mathrm{mg}$ of ephedrine and normal saline, $6 \mathrm{ml} / \mathrm{kg}$; the same doses were repeated as required. Bradycardia was treated by intravenous blouses of $0.5 \mathrm{mg}$ of atropine. And hypoxemia was treated with inhalation of pure oxygen through face mask. After the patient could communicate well at 60 minutes after surgery, the numerical pain rating scale (NRS) was used to assess patient's pain at rest and during coughing in the PACU. $100 \mathrm{mg}$ of intravenous tramadol was given as a rescue analgesic when the NRS was more than 3 at rest or during coughing. The tramadol consumption of every patient was recorded. Adverse events after the operation such as nausea, vomiting, hypotension, hypoxemia, cardiac arrhythmia, and other complications induced by drugs or puncture procedure of ESPB were all recorded and treated. The patients' total satisfaction with anesthesia regimen was evaluated verbally at 48 hours after surgery by a 5 -point Likert scale ( 5 = completely satisfied, 4 = quite satisfied, 3 = slightly dissatisfied, 2 = dissatisfied, 1 = very dissatisfied). All the data after surgery were recorded by an observer (W. S.J.) who was blind to the study design and group assignment.

Statistical analysis

The primary study endpoint was intraoperative sufentanil consumption. The dermatomal level of sensory block by ultrasound guided ESPB, pain score at 60 minutes after surgery, consumption of rescue analgesic tramadol, incidence of adverse events and patient satisfaction with anesthesia regimen were used as the secondary endpoint. Based on the result of previous study[8], excluding $0.2 \mu \mathrm{g} / \mathrm{kg}$ of sufentanil for induction, the average supplementary consumption of intraoperative sufentanil was 0.261 $\mu \mathrm{g} / \mathrm{kg} / \mathrm{h}$ for thoracic surgery. On the basis of preliminary experimental data, we calculated that a sample of 24 patients would provide $80 \%$ power at a two-sided alpha level of 0.05 . Ultimately, we recruited 30 patients in each group for a total of 60 patients considering possible dropouts.

The continuous variables were presented as means \pm standard deviation or median ( 25 th to 75 th centiles), and categorical data were presented as number and percentages. Normality was tested by the Kolmogorov-Smirnov analysis. For appropriate comparisons between the two groups, the chi-square test, 
Student t-test, Mann-Whitney U-test, or Fisher exact test was used if needed. All data were processed by IBM SPSS Statistics 21.0 (IBM Inc., New York, NY). A 2-sided p value less than 0.05 was considered to be statistically significant.

\section{Results}

Initially, sixty-two patients were recruited in this study. Two patients in group EG were excluded because they did not meet the inclusion criteria (one patient was known allergy to ropivacaine, the other patient was diagnosed with bradycardia). There were no significant differences in demographic and surgical data between the two groups (Table 1). Successful ultrasound guided ESPB was accomplished in all patients in group EG. At 30 minutes after the ESPB, the sensor block area in the left hemithorax was assessed in every patient in group EG. A sensory loss to pinprick from T3 to T9 over the entire posterolateral aspect of the left hemithorax, extending anteriorly to the midclavicular line was obtained in 16 patients. And a sensory block over the T2 to T8 dermatomes was obtained in 10 patients. There was an area of blocked sensation to pinprick extending from T2 to T9 in a cephalocaudad direction in 4 patients (Figure 3). Compared with group EG, the sufentanil consumption during the surgery was significantly higher in group GA (Table 2). The recovery time from anesthesia and the tracheal extubation time in group EG were significantly shorter than those in group GA (Table 2).

The pain score at rest or during coughing evaluated by NRS at 60 mins after the surgery in PACU was significantly lower in group EG compared with that in group GA (Table 2). Compared with group EG, the tramadol used for rescue analgesia in PACU was significantly more in group GA (Table 2). After the surgery, no respiratory depression, pruritus or uroschesis was observed in either group. And compared with group EG, the incidence of postoperative nausea and vomiting in group GA was much higher (Table 3). No complications related to ultrasound guided ESPB technique and local anesthetic toxicity were observed in the group EG. Compared with group GA, the patient satisfaction with anesthesia method was significantly higher in group EG (Table 3).

\section{Discussion}

This prospective, randomized, controlled clinical study was conducted to evaluate whether combined ultrasound guided ESPB and general anesthesia could provide a better anesthesia regimen compared with general anesthesia only in patients undergoing open thoracotomy. Ultrasound guided ESPB could provide an effective sensory block to pinprick in the operating area in patients undergoing open thoracotomy. And combined ultrasound guided ESPB with general anesthesia were found to have an opioid-sparing effect during surgery. In addition, compared with general anesthesia only, combined ultrasound guided ESPB with general anesthesia could provide a better patient satisfaction and induce less postoperative adverse events in patients undergoing open thoracotomy. 
As shown in the present study, ultrasound guided ESPB induced a sensory loss to pinprick from T2 to T9 in the left hemithorax. In a recent clinical report, Adhikary et al.[9] showed that $20 \mathrm{ml}$ of $0.5 \%$ ropivacaine injected via ESPB performed at T5 spinous process could provide sensory block between the T3 to T9 dermatomes, which was similar to the result of this study. The ESPB, first described by Forero $M$ et al. in 2016[6], was found effective in analgesia for thoracic neuropathic pain. As a previous cadaveric study found[6], at the exit from each intervertebral foramen, the upper thoracic spinal nerve split into a ventral and dorsal ramus. Then, passing through the costotransverse foramen, the dorsal ramus ran posteriorly and ascended into the deep plane of the erector spinae muscle. The ventral ramus extended laterally as the intercostal nerve and traveled deep to the internal intercostal membrane. Ultimately, the nerve branch which innervated the lateral and partial anterior thoracic wall arose from it. Based on the abovementioned cadaveric investigation, the ultrasound guided ESPB was performed successfully in this study. Previously, several studies found that $20 \mathrm{ml}$ of local anesthetics injected through ESPB could provide effective sensory block to pinprick from T2 to T9[6, 10, 11]. In order to cover enough dermatomes, $25 \mathrm{ml}$ of $0.5 \%$ ropivacaine was used in this study. In addition, in several previous clinical reports, the analgesic effect of ESPB was assessed only 20 minutes after the injection of ropivacaine[12, 13]. In this study, in order to make sure that the analgesic effect of ESPB has fully emerged, the sensory block to pinprick was assessed at 30 minutes after ropivacaine injection.

In this study, the intraoperative consumption of sufentanil in group EG was significantly less compared with that in group GA, which indicated that the ESPB could provide effective analgesia during the operation. Therefore, ESPB could provide a significant opioid-sparing effect in patients undergoing open thoracotomy. Although opioids were widely used for analgesia perioperatively, a recent study demonstrated that, the opioid dose was statistically significant negative correlated with the survival time in patients with pancreatic cancer[14]. Also, many other studies found that, high opioid administration during surgery increased the risk of cancer recurrence in patients with lung, prostate, larynx or oral cancer[15-20]. It was speculated that the $\mu$-opioid receptor induced cancer cell cycle progression, cancer cell proliferation and immunosuppression mediated these adverse effects on cancer treatment. Consequently, by the opioid-sparing effect, the ESPB probably provided potential benefits in cancer therapy for patients with esophageal cancer. In addition, as shown in this study, the pain scores at rest and during coughing at 60 minutes after surgery in group EG was significantly lower than those in group GA. Also, compared with group EG, the consumption of tramadol for rescue analgesia at 60 minutes after surgery was significantly higher in group GA. These results suggested that, at 30 minutes before the general anesthesia induction, $25 \mathrm{ml}$ of $0.5 \%$ ropivacaine injected for ESPB at T5 level could provide effect analgesia in the early postoperative period.

As found in this study, compared with group GA, the recovery time from general anesthesia and the tracheal extubation time were both shorter in group EG. This result suggested that the ESPB performed before general anesthesia contributed to provide a better quality of anesthesia recovery in patients undergoing open thoracotomy. Previous researches have demonstrated that accumulation of opioids resulted in delayed emergence from anesthesia[21, 22]. Based on this finding, the ESPB probably improved the quality of anesthesia recovery by reducing the intraoperative consumption of sufentanil. In 
the present study, the incidence of postoperative nausea and vomiting in group EG was significantly lower than that in group GA, which indicated that ESPB could markedly reduce the incidence of adverse events related to general anesthesia. Previous research has demonstrated that opioids induced nausea and vomiting by activating opioid receptors in the chemoreceptor trigger zone, vestibular apparatus and gastrointestinal tract[23]. And the severity degree of nausea and vomiting was positively correlated to the consumption of opioids $[23,24]$. Therefore, the ESPB probably reduced the incidence of nausea and vomiting by decreasing the consumption of sufentanil during surgery. At last, in this study, compared with group GA, the patient satisfaction with anesthesia regimen was significantly higher in group EG, which was consistent with higher quality of anesthesia recovery, lower pain score, less incidence of nausea and vomiting in group EG.

There were several limitations in the present clinical study. First, according to the results of previous studies, only one volume and one concentration of ropivacaine was used for ultrasound guided ESPB in this study. However, a volume-response or concentration-response study was not performed to obtain the optimal volume and concentration of ropivacaine used in ultrasound guided ESPB for open thoracotomy in order to get a better analgesic effect and less adverse events. Second, in this study, only one single bolus of ropivacaine was injected for ultrasound guided ESPB. However, continuous infusion of ropivacaine for ESPB was not performed in this study. Thus, whether continuous infusion of local anesthetic for ESPB could provide longer postoperative analgesia and better patient satisfaction was not clear. Third, this study was a single center clinical study. The sample size was relatively small. And patients recruited in this study were relatively healthy (American Society of Anesthesiologists I and II). More patients with or without comorbidities need to be recruited in future multi-center clinical studies.

\section{Conclusions}

In conclusion, this study demonstrated that, compared with general anesthesia only, combined ultrasound guided ESPB and general anesthesia could significantly reduce intraoperative sufentanil consumption, improve quality of anesthesia recovery, reduce incidence of postoperative nausea and vomiting and improve satisfaction in patients undergoing open thoracotomy. Thus, combined ultrasound guided ESPB with general anesthesia was a recommended multi-mode anesthesia regimen for open thoracotomy.

\section{Abbreviations}

ASA: American Society of Anesthesiologists; bpm: Beats per minute; EG: Combined ultrasound guided erector spinae plane block and general anesthesia; ESM: Erector spinae muscle; ESPB: Erector spinae plane block; GA: General anesthesia; MAC: Minimum alveolar concentration; NRS: Numerical pain rating scale; PACU: Postanesthesia care unit; RMM: Rhomboid major; $\mathrm{SpO}_{2}$ : Pulse oxygen saturation; TM: Trapezius; TP: Transverse process; T2, T3, T4, T5, T6, T7, T8, T9: Second thoracic vertebra, Third thoracic vertebra, Fourth thoracic vertebra, Fifth thoracic vertebra, Sixth thoracic vertebra, Seventh thoracic vertebra, Eighth thoracic vertebra, Ninth thoracic vertebra 


\section{Declarations}

\section{Acknowledgements}

We acknowledge the department of anesthesiology team and the department of thoracic surgery team in National Cancer Center/National Clinical Research Center for Cancer/Cancer Hospital, Chinese Academy of Medical Sciences and Peking Union Medical College for supporting the research.

\section{Funding}

This study was funded by the Beijing Hope Run Special Fund of Cancer Foundation of China (grant LC2017A09). The funding had no role in study design, data collection and analysis, decision to publish or preparation of the manuscript.

\section{Availability of data and materials}

The datasets used and/or analyzed during the current study are available from the co-corresponding authors on reasonable request.

\section{Authors' contributions}

QW contributes to research design, data collection, data analysis and paper writing. SL contributes to data collection. SW contributes to data collection. GZ contributes to research design, data analysis and paper writing. YL contribute to data collection. $\mathrm{HZ}$ contributes to research design, data analysis and paper writing. All authors have read and approved the final manuscript.

\section{Ethics approval and consent to participate}

This study was approved by the institutional ethics committee of National Cancer Center/National Clinical Research Center for Cancer/Cancer Hospital, Chinese Academy of Medical Sciences and Peking Union Medical College (NCC201804009), and registered on ClinicalTrials.gov (ChiCTR1800016583). The written informed consent was obtained from every patient.

\section{Consent for publication}

Not applicable. 


\section{Competing interests}

The authors declare that they have no competing interests.

\section{Author details}

${ }^{1}$ Department of Anesthesiology, National Cancer Center/National Clinical Research Center for Cancer/Cancer Hospital, Chinese Academy of Medical Sciences and Peking Union Medical College, No. 17 Panjiayuannanli, Chaoyang District, Beijing, China. ${ }^{2}$ Department of thoracic surgery, National Cancer Center/National Clinical Research Center for Cancer/Cancer Hospital, Chinese Academy of Medical Sciences and Peking Union Medical College, No. 17 Panjiayuannanli, Chaoyang District, Beijing, China.

\section{References}

1. Fornasari D. Pain pharmacology: focus on opioids. Clin Cases Miner Bone Metab. 2014;11(3):165-8.

2. Manchikanti L, Singh A. Therapeutic opioids: a ten-year perspective on the complexities and complications of the escalating use, abuse, and nonmedical use of opioids. Pain Physician. 2008;11(2 Suppl):S63-88.

3. Crumley S, Schraag S. The role of local anaesthetic techniques in ERAS protocols for thoracic surgery. J Thorac Dis. 2018;10(3):1998-2004.

4. Cook TM, Counsell D, Wildsmith JA. Major complications of central neuraxial block: report on the Third National Audit Project of the Royal College of Anaesthetists. Br J Anaesth. 2009;102(2):179-90.

5. D'Ercole F, Arora H, Kumar P A. Paravertebral Block for Thoracic Surgery. J Cardiothorac Vasc Anesth. 2018;32(2):915-27.

6. Forero M, Adhikary SD, Lopez H, Tsui C, Chin KJ. The Erector Spinae Plane Block: A Novel Analgesic Technique in Thoracic Neuropathic Pain. Reg Anesth Pain Med. 2016;41(5):621-7.

7. Adhikary SD, Pruett A, Forero M, Thiruvenkatarajan V. Erector spinae plane block as an alternative to epidural analgesia for post-operative analgesia following video-assisted thoracoscopic surgery: A case study and a literature review on the spread of local anaesthetic in the erector spinae plane. Indian J Anaesth. 2018;62(1):75-8.

8. Rosseel PM, van den Broek WG, Boer EC, Prakash O. Epidural sufentanil for intra- and postoperative analgesia in thoracic surgery: a comparative study with intravenous sufentanil. Acta Anaesthesiol Scand. 1988;32(3):193-8.

9. Adhikary SD, Prasad A, Soleimani B, Chin KJ. Continuous Erector Spinae Plane Block as an Effective Analgesic Option in Anticoagulated Patients After Left Ventricular Assist Device Implantation: A Case 
Series. J Cardiothorac Vasc Anesth. 2018;Apr 12.pii:S1053-0770. [Epub ahead of print]

10. Hamilton DL, Manickam B. Erector spinae plane block for pain relief in rib fractures. $\mathrm{Br} \mathrm{J}$ Anaesth. 2017;118(3):474-5.

11. Veiga M, Costa D, Brazão I. Erector spinae plane block for radical mastectomy: A new indication? Rev Esp Anestesiol Reanim. 2018;65(2):112-5.

12. Forero $M$, Rajarathinam $M$, Adhikary $S$, Chin KJ. Erector spinae plane (ESP) block in the management of post thoracotomy pain syndrome: A case series. Scand J Pain. 2017;17:325-9.

13. Kimachi PP, Martins EG, Peng P, Forero M. The Erector Spinae Plane Block Provides Complete Surgical Anesthesia in Breast Surgery: A Case Report. A A Pract. 2018;11(7):186-8.

14. Oh TK, Do SH, Yoon YS, Song IA. Association Between Opioid Use and Survival Time in Patients With Unresectable Pancreatic Cancer: 10 Years of Clinical Experience. Pancreas. 2018;47(7):837-42.

15. Cata JP, Keerty V, Keerty D, Feng L, Norman PH, Gottumukkala V, Mehran JR, Engle M. A retrospective analysis of the effect of intraoperative opioid dose on cancer recurrence after non-small cell lung cancer resection. Cancer Med. 2014;3(4):900-8.

16. Cata JP, Zafereo M, Villarreal J, Unruh BD, Truong A, Truong DT, Feng L, Gottumukkala V. Intraoperative opioids use for laryngeal squamous cell carcinoma surgery and recurrence: a retrospective study. J Clin Anesth. 2015;27(8):672-9.

17. Patino MA, Ramirez RE, Perez CA, Feng L, Kataria P, Myers J, Cata JP. The impact of intraoperative opioid use on survival after oral cancer surgery. Oral Oncol. 2017;74:1-7.

18. Zylla D, Gourley BL, Vang D, Jackson S, Boatman S, Lindgren B, Kuskowski MA, Le C, Gupta K, Gupta P. Opioid requirement, opioid receptor expression, and clinical outcomes in patients with advanced prostate cancer. Cancer. 2013;119(23):4103-10.

19. Zylla D, Kuskowski MA, Gupta K, Gupta P. Association of opioid requirement and cancer pain with survival in advanced non-small cell lung cancer. Br J Anaesth. 2014;113(Suppl 1):i109-16.

20. Forget $P$, Tombal B, Scholtès JL, Nzimbala J, Meulders C, Legrand C, Van Cangh P, Cosyns JP, De Kock M. Do intraoperative analgesics influence oncological outcomes after radical prostatectomy for prostate cancer? Eur J Anaesthesiol. 2011;28(12):830-5.

21. Caspi J, Klausner JM, Safadi T, Amar R, Rozin RR, Merin G. Delayed respiratory depression following fentanyl anesthesia for cardiac surgery. Crit Care Med. 1988;16(3):238-40.

22. Helmers JH, Van Leeuwen L, Adam A, Giezen J, Deen L. Double-blind comparison of the postoperative respiratory depressant effects of alfentanil and fentanyl. Acta Anaesthesiol Belg. 1982;33(1):13-21.

23. Nicholson BD. Economic and clinical burden of opioid-induced nausea and vomiting. Postgrad Med. 2017;129(1):111-7.

24. Smith HS, Laufer A. Opioid induced nausea and vomiting. Eur J Pharmacol. 2014;722:67-78.

\section{Tables}


Table 1 Demographic and Surgical Data

\begin{tabular}{lccc}
\hline & Group EG & Group GA & $p$-Value \\
& $(n=30)$ & $(n=30)$ & \\
\hline Sex (male/female) & $18 / 12$ & $17 / 13$ & 0.793 \\
Age (year) & $57.6 \pm 0.62$ & $56.9 \pm 0.68$ & 0.499 \\
Weight (kg) & $64.8 \pm 3.53$ & $64.1 \pm 4.25$ & 0.470 \\
Height (cm) & $169.5 \pm 3.99$ & $167.9 \pm 3.65$ & 0.103 \\
ASA (I/II) & $13 / 17$ & $15 / 15$ & 0.796 \\
Duration of surgery (min) & $150.9 \pm 3.76$ & $152.9 \pm 5.62$ & 0.123 \\
\hline
\end{tabular}

ASA: American Society of Anesthesiologists

Table 2 Anesthetic and Analgesic Data

\begin{tabular}{lccc}
\hline & Group EG & Group GA & $p$-Value \\
& $(n=30)$ & $(n=30)$ & \\
\hline Duration of anesthesia (min) & $179.5 \pm 4.01$ & $192.1 \pm 3.66$ & $<0.001$ \\
Recovery time (min) & $12.2 \pm 2.82$ & $22.3 \pm 3.65$ & $<0.001$ \\
Tracheal extubation time (min) & $13.7 \pm 2.9$ & $24.7 \pm 3.6$ & $<0.001$ \\
Intraoperative sufentanil consumption (ug/kg/h) & $0.166 \pm 0.016$ & $0.274 \pm 0.019$ & $<0.001$ \\
Pain score at rest (numerical) & $1.9 \pm 1.2$ & $3.6 \pm 1.4$ & $<0.001$ \\
Pain score during coughing (numerical) & $2.1 \pm 1.2$ & $3.8 \pm 1.5$ & $<0.001$ \\
Tramadol usage (Used/Not used) & $3 / 27$ & $15 / 15$ & 0.001 \\
\hline
\end{tabular}

Table 3 Adverse Events and Patient Satisfaction 


\begin{tabular}{lcccc}
\hline & Group EG & Group GA & $p$-Value \\
& $(n=30)$ & $(n=30)$ & \\
\hline Nausea and vomiting & $2(6.7 \%)$ & $10(33.3 \%)$ & 0.021 \\
Respiratory depression & 0 & 0 & - \\
pruritus & & 0 & 0 & - \\
uroschesis & & 0 & 0 & - \\
Patient satisfaction (5-point Likert scale) & 1 & 0 & $2(6.7 \%)$ & $<0.001$ \\
& 2 & 0 & $4(13.3 \%)$ & \\
& 3 & $8(26.7 \%)$ & $18(60 \%)$ & \\
& 4 & $20(66.7 \%)$ & $6(20 \%)$ & \\
& 5 & $2(6.7 \%)$ & 0 & \\
\hline
\end{tabular}

\section{Figures}

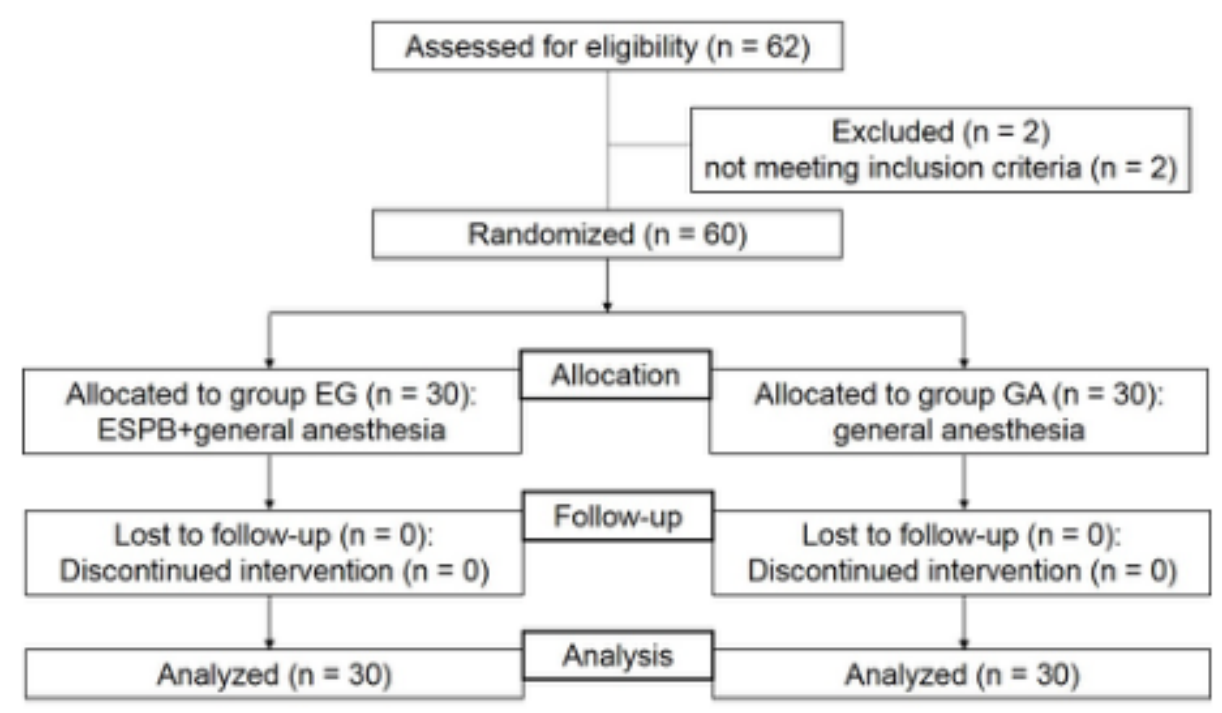

Figure 1

CONSORT flow diagram of this study. A total of 62 patients were enrolled in this study. Two patients in group EG were excluded because they did not meet the inclusion criteria (one patient was known allergy to ropivacaine, the other patient was diagnosed with bradycardia). Therefore, 30 patients in each group had completed the study. ESPB: erector spinae plane block 


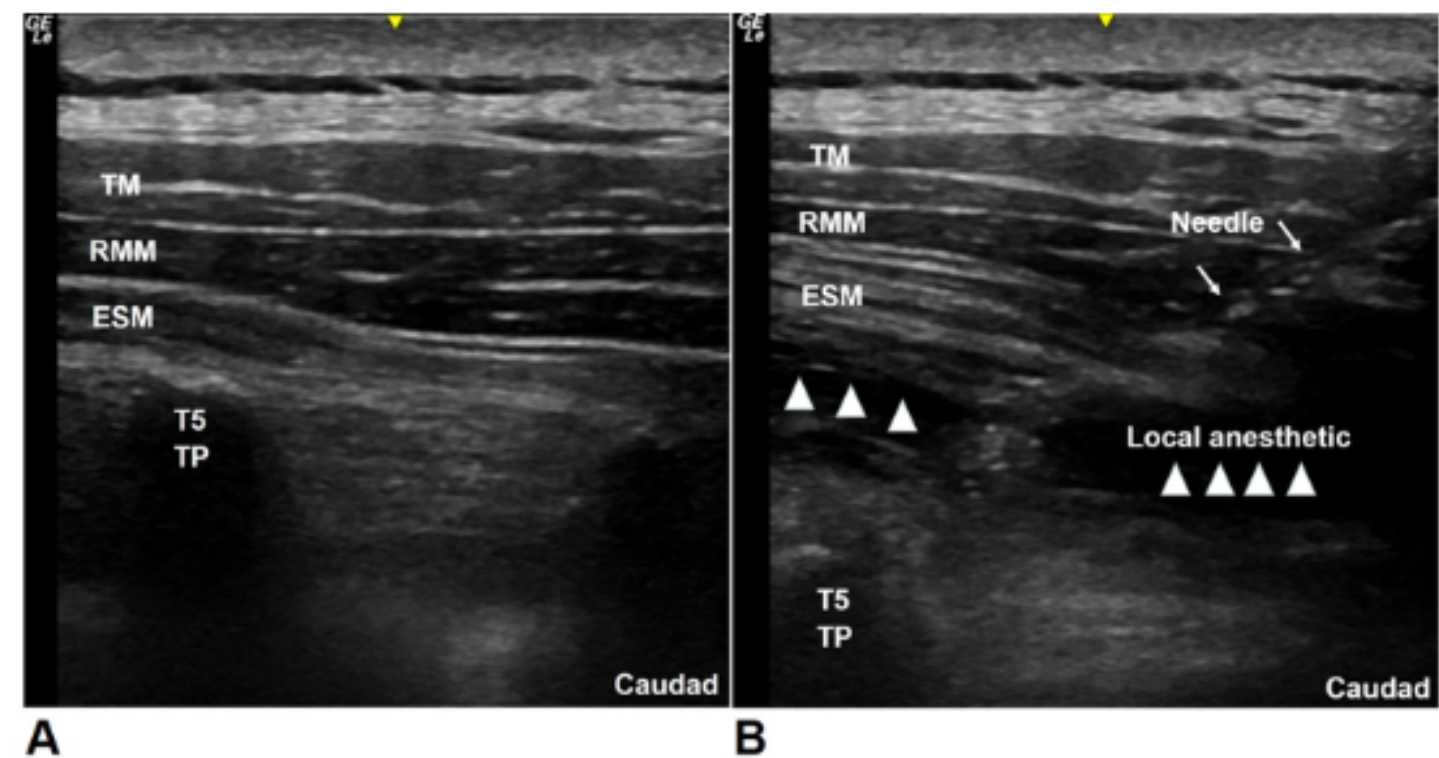

\section{Figure 2}

The ultrasound guided ESPB performed deep to the erector spinae muscle (ESM) in a patient in the right lateral decubitus position. A, Ultrasound imaging of the ESM at the level of the fifth thoracic vertebra. B, Injection of ropivacaine into the interfascial plane deep to ESM produced a visible fluid spread (white solid triangles) beneath the ESM. TM: trapezius RMM: rhomboid major ESM: erector spinae muscle TP: transverse process $\mathrm{T} 5$ : the fifth thoracic vertebra

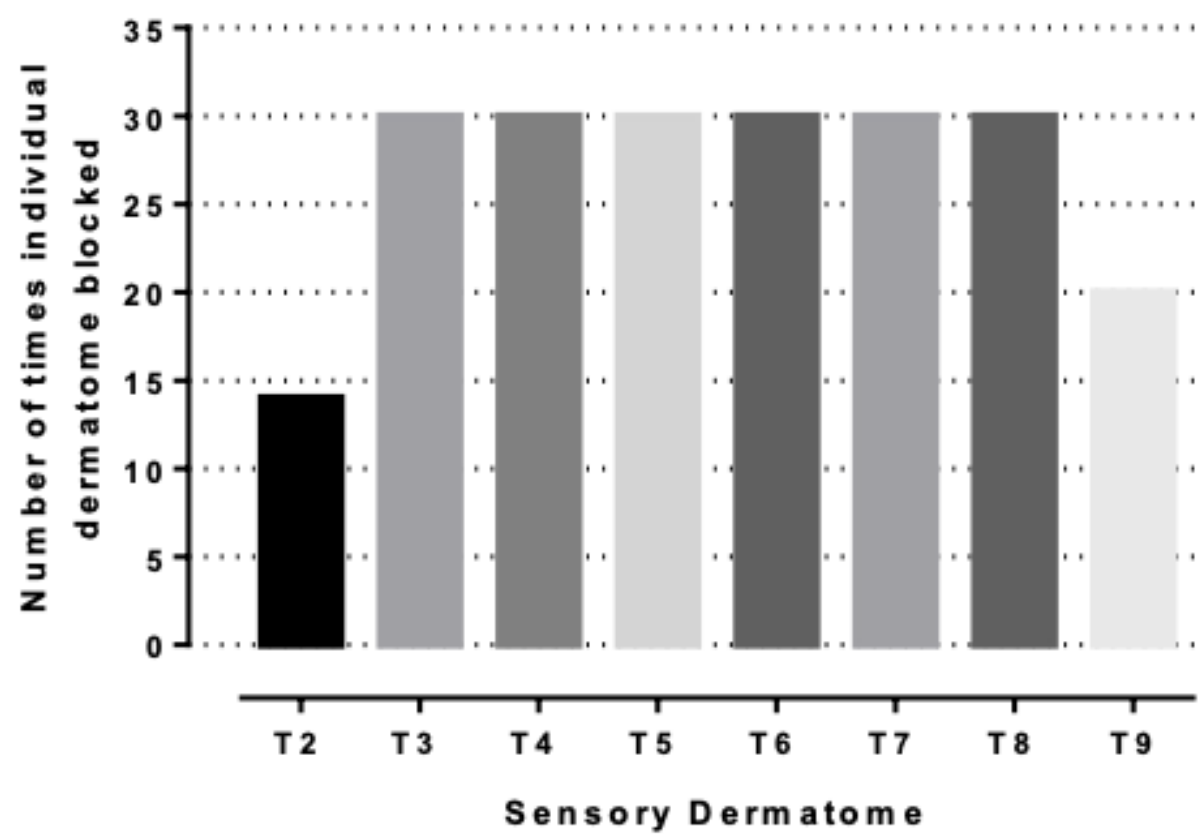

\section{Figure 3}

Bar graph showing the number of times each individual sensory dermatome was blocked ( $\mathrm{Y}$-axis) and $\mathrm{X}$ axis represents sensory dermatomes range from T2-T9. T2, T3, T4, T5, T6, T7, T8, T9: Second thoracic 
vertebra, Third thoracic vertebra, Fourth thoracic vertebra, Fifth thoracic vertebra, Sixth thoracic vertebra, Seventh thoracic vertebra, Eighth thoracic vertebra, Ninth thoracic vertebra 